\section{Caracterização epidemiológica dos casos de AIDS em pessoas com 60 anos ou mais, Pernambuco, Brasil, 1998 a 2008}

\author{
Epidemiological characteristics of AIDS cases in \\ persons aged 60 years or older, Pernambuco State, \\ Brazil, 1998 to 2008
}

\section{Caracterización epidemiológica de casos de SIDA en personas mayores de 60 años o más, estado de Pernambuco, Brasil, de 1998 a 2008}

\author{
1 Centro de Pesquisas Aggeu \\ Magalhães, Fundação \\ Oswaldo Cruz, Recife, Brasil. \\ 2 Prefeitura da Cidade do \\ Recife, Recife, Brasil. \\ Correspondência \\ M. M. Silva \\ Centro de Pesquisas Aggeu \\ Magalhães, Fundação Oswaldo \\ Cruz. \\ Rua Newton Braga 118, \\ Olinda, PE 53110-020, Brasil. \\ marcellaonline@ig.com.br
}

\begin{abstract}
Changes have occurred in the world scenario in recent years due to declining fertility and mortality, and longevity has thus appeared as a real phenomenon. Sexuality of the elderly is viewed with various prejudices. The current study characterized AIDS cases in persons 60 years or older in Pernambuco State, Brazil, reported to the State Health Department from January 1, 1998, to December 31, 2008. A cross-sectional descriptive study was performed with data from the Information System on Diseases of Notification (SINAN). In 1998 the AIDS incidence rate in the target age bracket was 1.6 cases per 100 thousand, increasing to 4.8 per 100 thousand in 2008, or an increase of $200 \%$. There is thus an evident need to develop measures for prevention, diagnosis, and patient care specifically targeting the elderly, since sexuality in this age bracket is still enshrouded by myths and taboos.
\end{abstract}

HIV; Acquired Immunodeficiency Syndrome; Demographic Aging
Marcella Monteiro da Silva 1

Ana Lúcia Ribeiro de Vasconcelos 1 Leila Karina de Novaes P. Ribeiro 2

\section{Resumo}

Mudanças vêm acontecendo no panorama mundial nos últimos anos devido ao declínio das taxas de fertilidade e mortalidade, e como decorrência a longevidade tem sido apresentada como um fenômeno real. Na maturidade, a sexualidade vem acrescida de vários preconceitos. O presente trabalho caracterizou os casos de AIDS em pessoas com 60 anos ou mais, residentes no Estado de Pernambuco, Brasil, notificados à Secretaria de Saúde de Pernambuco entre 1o de janeiro de 1998 e 31 de dezembro de 2008. Foi realizado um estudo transversal/descritivo, com base nos dados de notificação do Sistema de Informação de Agravos de Notificação (SINAN). Em 1998 a taxa de incidência de AIDS, na população de estudo, era de 1,6 caso/100 mil habitantes, passando para 4,8 casos/100 mil habitantes em 2008, caracterizando um aumento de $200 \%$. Fica evidente a necessidade de desenvolvimento de ações de prevenção, diagnóstico e assistência direcionada especificamente para a terceira idade, uma vez que a sexualidade nesta fase da vida ainda é envolta por muitos tabus e mitos.

HIV; Síndrome de Imunodeficiência Adquirida; Envelhecimento da População 


\section{Introdução}

A infecção pelo HIV constitui um fenômeno global, dinâmico e instável, dependente, dentre outros, do comportamento individual e coletivo ${ }^{1}$.

Recordando, os primeiros casos de AIDS datam de 1977-1978, nos Estados Unidos, Haiti e África Central, concentrados nos grandes centros urbanos, acometendo principalmente homossexuais. Gradativamente atingiu outros grupos populacionais: usuários de drogas injetáveis (UDI), indivíduos expostos a sangue e hemoderivados, mulheres e crianças 2 .

No Brasil, os primeiros casos de AIDS notificados datam de 1982, localizado no eixo Rio de Janeiro-São Paulo, sugerindo ter sido a Região Sudeste o foco inicial dessa epidemia 3 .

No período entre 1982-2008, o Ministério da Saúde registrou 465 mil casos novos de AIDS no Brasil, sendo a maioria (61\%) na Região Sudeste 4 .

Em Pernambuco, a epidemia de AIDS teve início em 1983, com o primeiro caso identificado no Hospital das Clínicas da Universidade Federal de Pernambuco. Desde então, apresenta tendências crescentes, chegando a 533 casos em 1990 e a 5.395 no ano 20003.

Assim, o presente estudo teve por objetivo caracterizar os casos de AIDS em pessoas com 60 anos ou mais, residentes em Pernambuco, entre 1o de janeiro de 1998 e 31 de dezembro de 2008.

\section{Metodologia}

Trata-se de um estudo transversal, descritivo, realizado com base nos dados do Sistema de Informações sobre Agravos de Notificação (SINAN), do Núcleo Estadual de Epidemiologia da Secretaria Estadual de Saúde de Pernambuco. A população estudada foi de pessoas com 60 anos ou mais, residentes em Pernambuco, diagnosticadas com AIDS entre 1998-2008.

Os dados populacionais utilizados foram provenientes de censos demográficos ou projeções e estimativas do Instituto Brasileiro de Geografia e Estatística (IBGE; http://www.ibge.gov.br), acessados por meio eletrônico.

As variáveis analisadas foram: faixa etária, sexo, raça/cor, grau de escolaridade (anos de estudos), categoria de exposição, município de residência e ano do diagnóstico, sendo demonstrada a frequência e distribuição dos casos.

Com base nos municípios de residência os casos foram agrupados e demonstrados segundo as regionais de saúde do estado. Foram calculadas as taxas de incidência de AIDS na população do estudo, por ano de diagnóstico.

\section{Resultados}

Tem-se na Tabela 1 o número de casos de AIDS na população do trabalho, notificados segundo as Gerências Regionais de Saúde (GERES). Pode-se observar um total de 293 casos, e que 183 $(62,5 \%)$ residiam na I GERES. Verifica-se ainda, que apenas essa regional notificou casos em todos os anos analisados, caracterizando indícios de subnotificação - não houve notificação na XI GERES.

Está na Figura 1 a constatação de crescimento de $200 \%$ do número de casos notificados - incidência de 1,6 caso/100 mil habitantes em 1998 e 4,8 casos / 100 mil habitantes em 2008.

As características epidemiológicas estão demonstradas na Tabela 2, evidenciando que 195 casos $(66,5 \%)$ foram em homens. O grupo etário de 60 a 69 anos foi o mais acometido (79,5\%). Quanto à escolaridade, os analfabetos são quase 8\%, demonstrando igual proporção com 12 anos ou mais de estudos. Pode-se observar que predomina a baixa escolaridade: 82 casos $(53,95 \%)$ com escolaridade menor que oito anos de estudos e $23(15,13 \%)$ analfabetos, que somados totalizam 105 casos (69,08\%). Demonstra-se, ainda, que a maioria dos casos - $131(44,7 \%)$ - foi da categoria heterossexual, e que os homens que fazem sexo com outros homens (HSH) corresponderam a 32 casos $(10,9 \%)$.

Observou-se que não houve nenhum caso em mulheres nas categorias homo/bissexual; tendose dentre os classificados como heterossexual 58 homens (19,8\%) e 73 mulheres (24,9\%). Dos 130 casos com essa informação ignorada, 105 (35,9\%) foram em homens e 25 (8,5\%) em mulheres.

Quanto à variável raça/cor, 67 casos (22,9\%) foram classificados como brancos; 104 (35,5\%) como pardos ou pretos; 01 ( $0,3 \%)$ como amarelo; e 121 casos (41,3\%) como "ignorados".

\section{Discussão}

A epidemia da AIDS tem hoje no critério vulnerabilidade seu maior foco. No Brasil e no mundo os dados epidemiológicos demonstram que a epidemia avançou acometendo grupos sociais mais vulneráveis, estando os idosos entre estes 1,2. A expansão da AIDS, apesar das campanhas preventivas, é apontada em pesquisas como consequência de mudanças comportamentais impulsionadas pelo fenômeno da globalização, da massificação da notícia e da relativização de valores 5,6. É dito, ainda, que o aumento da infecção em idosos se deve a não assimilação/ adoção por parte desta população das medidas para o sexo seguro. Atualmente, medicamentos 
Tabela 1

Distribuição dos casos de AIDS em pessoas com 60 anos ou mais, segundo as Gerências Regionais de Saúde (GERES) de Pernambuco, Brasil, 1998 a 2008

\begin{tabular}{lcccccccccccccc}
\hline GERES & 1998 & 1999 & 2000 & 2001 & 2002 & $\mathbf{2 0 0 3}$ & $\mathbf{2 0 0 4}$ & $\mathbf{2 0 0 5}$ & $\mathbf{2 0 0 6}$ & $\mathbf{2 0 0 7}$ & $\mathbf{2 0 0 8}$ & Total & $\%$ \\
\hline I & 12 & 11 & 9 & 15 & 21 & 15 & 20 & 27 & 18 & 15 & 20 & 183 & 62,5 \\
II & 0 & 0 & 0 & 0 & 0 & 1 & 0 & 0 & 1 & 0 & 0 & 2 & 0,7 \\
III & 0 & 1 & 1 & 0 & 0 & 0 & 0 & 0 & 1 & 0 & 0 & 3 & 1,0 \\
IV & 0 & 1 & 1 & 0 & 3 & 1 & 0 & 3 & 2 & 4 & 2 & 17 & 5,8 \\
V & 0 & 0 & 0 & 1 & 1 & 0 & 0 & 0 & 1 & 0 & 0 & 3 & 1,0 \\
VI & 0 & 0 & 0 & 0 & 0 & 0 & 0 & 1 & 0 & 0 & 0 & 1 & 0,3 \\
VII & 0 & 0 & 0 & 0 & 0 & 0 & 0 & 1 & 0 & 0 & 0 & 1 & 0,3 \\
VIII & 0 & 1 & 0 & 0 & 0 & 0 & 0 & 0 & 1 & 0 & 1 & 3 & 1,0 \\
IX & 0 & 0 & 0 & 0 & 1 & 0 & 0 & 0 & 1 & 0 & 0 & 2 & 0,7 \\
X & 0 & 0 & 1 & 0 & 0 & 0 & 0 & 0 & 0 & 0 & 0 & 1 & 0,3 \\
XI & 0 & 0 & 0 & 0 & 0 & 0 & 0 & 0 & 0 & 0 & 0 & 0 & 0,0 \\
Pernambuco & 12 & 14 & 12 & 16 & 26 & 17 & 20 & 32 & 25 & 19 & 23 & 216 & 73,7 \\
Ignorado & 0 & 0 & 9 & 8 & 10 & 7 & 7 & 2 & 15 & 19 & 0 & 77 & 26,3 \\
Total & 12 & 14 & 21 & 24 & 36 & 24 & 27 & 34 & 40 & 38 & 23 & 293 & 100,0 \\
\hline
\end{tabular}

Fonte: Sistema de Informação de Agravos de Notificação (SINAN), 2010.

Figura 1

Incidência dos casos de AIDS por 100 mil habitantes em pessoas com 60 anos ou mais, segundo ano de diagnóstico.

Pernambuco, Brasil, 1998 a 2008.

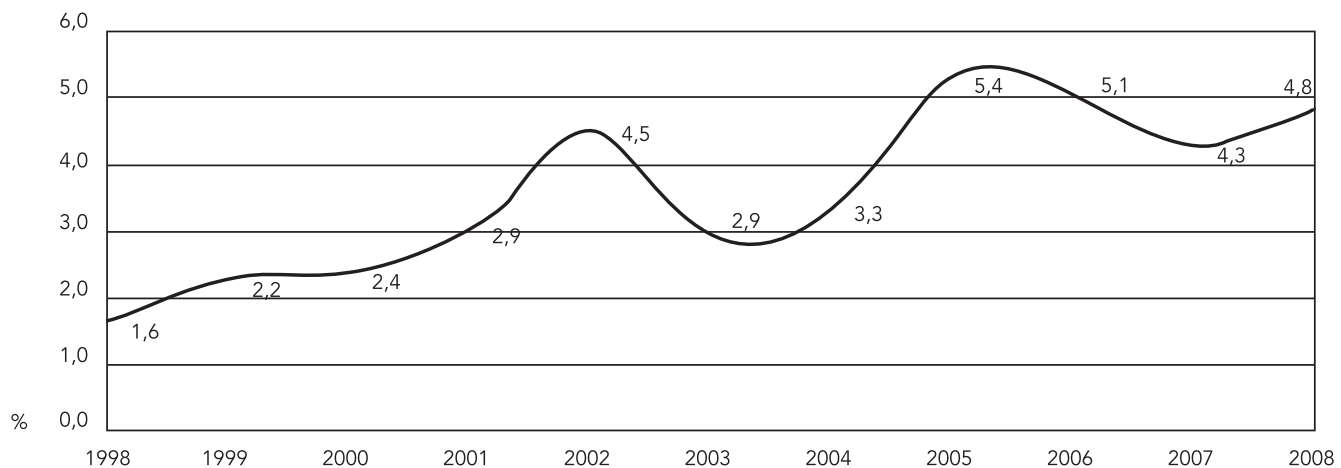

Fonte: Sistema de Informação de Agravos de Notificação (SINAN), 2010.

que inibem a impotência sexual e a reposição hormonal fazem com que os idosos passem a ter uma vida sexual mais ativa, não utilizando, contudo, medidas preventivas por não se sentirem vulneráveis 6 .

Pesquisas brasileiras constatam o aumento de casos de AIDS em pessoas com menor escolaridade 7,8,9,10,11, ficando esta condição demonstrada neste estudo.
A via heterossexual foi a categoria de maior expressão, reforçando que a prática sexual, sem proteção, constitui a mais importante via de transmissão também entre os idosos, denotando a necessidade de abordagens para o sexo seguro também para esta faixa etária.

Chama a atenção o fato de não ter havido nenhum caso de UDI entre os idosos deste trabalho. Possivelmente, assumindo erroneamente que es- 
Distribuição dos casos de AIDS em pessoas com 60 anos ou mais, segundo sexo, anos de estudos e categoria de exposição. Pernambuco, Brasil, 1998 a 2008.

\begin{tabular}{|c|c|c|c|c|c|c|c|c|}
\hline \multirow[t]{3}{*}{ Variáveis } & \multicolumn{8}{|c|}{ Faixa etária (anos) } \\
\hline & \multicolumn{2}{|c|}{$60-69$} & \multicolumn{2}{|c|}{$70-79$} & \multicolumn{2}{|c|}{80 e mais } & \multicolumn{2}{|c|}{ Total } \\
\hline & $\mathrm{n}$ & $\%$ & $\mathrm{n}$ & $\%$ & $\mathbf{n}$ & $\%$ & $\mathbf{n}$ & $\%$ \\
\hline \multicolumn{9}{|l|}{ Sexo } \\
\hline Masculino & 159 & 54,2 & 33 & 11,3 & 3 & 1,0 & 195 & 66,5 \\
\hline Feminino & 74 & 25,3 & 18 & 6,2 & 6 & 2,0 & 98 & 33,5 \\
\hline \multicolumn{9}{|l|}{ Anos de estudos } \\
\hline Nenhum & 19 & 6,4 & 3 & 1,0 & 1 & 0,3 & 23 & 7,8 \\
\hline $1-3$ & 38 & 13,0 & 6 & 2,1 & 1 & 0,3 & 45 & 15,4 \\
\hline $4-7$ & 28 & 9,5 & 7 & 2,4 & 2 & 0,7 & 37 & 12,6 \\
\hline $8-11$ & 21 & 7,1 & 2 & 0,7 & 0 & 0,0 & 23 & 7,8 \\
\hline 12 e mais & 20 & 6,8 & 3 & 1,0 & 1 & 0,3 & 24 & 8,2 \\
\hline Ignorado & 107 & 36,5 & 30 & 10,2 & 4 & 1,4 & 141 & 48,1 \\
\hline \multicolumn{9}{|c|}{ Categoria de exposição } \\
\hline Homossexual & 15 & 5,1 & 3 & 1,0 & 2 & 0,7 & 20 & 6,8 \\
\hline Bissexual & 10 & 3,4 & 2 & 0,7 & 0 & 0,0 & 12 & 4,1 \\
\hline Heterossexual & 108 & 36,9 & 19 & 6,5 & 4 & 1,4 & 131 & 44,7 \\
\hline UDI & 0 & 0,0 & 0 & 0,0 & 0 & 0,0 & 0 & 0,0 \\
\hline Ignorado & 100 & 34,2 & 27 & 9,2 & 3 & 1,0 & 130 & 44,4 \\
\hline Total & 233 & 79,5 & 51 & 17,4 & 9 & 3,1 & 293 & 100,0 \\
\hline
\end{tabular}

UDI: usuários de drogas injetáveis.

Fonte: Sistema de Informação de Agravos de Notificação (SINAN), 2010

se grupo não é passível de tal comportamento, os profissionais de saúde não tenham investigado essa prática (no presente ou no passado) nessa população, embora seja essa abordagem um fator importante na investigação epidemiológica do caso 10,11 .

A ausência de casos em alguns anos deste estudo pode refletir a falta de diagnóstico, a subnotificação, atraso na investigação, denotando, todavia, baixa qualidade da informação coletada, sendo este um dos principais problemas da vigilância epidemiológica 12 .

\section{Considerações finais}

O envelhecimento para ser experiência positiva exige independência e qualidade de vida, concebendo o exercício da sexualidade como natural e, portanto, inerente a essa população.

A incidência de AIDS na população estudada faz supor que as campanhas de prevenção sobre HIV/AIDS e outras DST, até então realizadas, não estão atingindo eficazmente essa população. Faz-se necessário que campanhas específicas sejam pensadas e que os serviços de saúde abordem essa temática durante as consultas de rotina em atendimento aos idosos, possibilitando a prevenção primária. Para isso, é preciso capacitar profissionais, não só os envolvidos com a vigilância epidemiológica, mas, especificamente, os que assistem aos pacientes, para que atentem sobre a importância da informação.

A confiabilidade, completude e atualização dos dados de notificação melhoram a qualidade e privilegiarão as tomadas de decisões. 


\section{Resumen}

Se están produciendo cambios en el escenario mundial durante los últimos años, debido a la reducción de las tasas de fecundidad y mortalidad, y como resultado, la longevidad se presenta como un fenómeno real. Durante la madurez, la sexualidad viene asociada a prejuicios más diversos. Este estudio caracteriza los casos de SIDA en personas de 60 años o más que vivían en el estado de Pernambuco, Brasil, y que se notificaron al Departamento de Salud de Pernambuco entre el 1 enero 1998 al 31 diciembre 2008. Se realizó un estudio transversal/ datos descriptivos con información del Sistema de Notificación de Resultados. En 1998, la tasa de incidencia del SIDA en la población estudiada fue de 1,6 casos por cada 100 mil habitantes, elevándose a 4,8 casos/100 mil habitantes en 2008, con un incremento del 200\%. Es evidente la necesidad de desarrollo de la prevención, el diagnóstico y la asistencia dirigida específicamente a las personas mayores, ya que la sexualidad esa etapa de la vida, sigue rodeada de muchos tabúes y mitos.

VIH; Síndrome de Inmunodeficiencia Adquirida; Envejecimiento de la Población

\section{Colaboradores}

M. M. Silva, A. L. R. Vasconcelos e L. K. N. P. Ribeiro contribuíram na concepção do desenho, análise e interpretação dos dados, redação do artigo, e elaboraram e fizeram todas as correções necessárias até a aprovação da versão final.

\section{Agradecimentos}

Agradecemos ao Departamento de Saúde Coletiva, Centro de Pesquisas Aggeu Magalhães, Fundação Oswaldo Cruz e ao Núcleo de Epidemiologia da Secretaria Estadual de Saúde de Pernambuco, pelo incentivo à pesquisa e ao fornecimento de dados.

\section{Referências}

1. Brito A, Castilho E, Szwarcwald C. AIDS e infecção pelo HIV no Brasil: uma epidemia multifacetada. Rev Soc Bras Med Trop 2001; 34:207-17.

2. Ministério da Saúde. Curso básico de vigilância epidemiológica. Brasília: Ministério da Saúde; 2005.

3. Barbosa LM. A dinâmica da epidemia de AIDS nas regiões nordeste e sudeste [Tese de Doutorado]. Belo Horizonte: Universidade Federal de Minas Gerais; 2001.

4. Rede Interagencial de Informação para a Saúde. Indicadores básicos para a saúde no Brasil: conceitos e aplicações. 2a Ed. Brasília: Organização PanAmericana da Saúde; 2008.

5. Grangeiro A, Escuder M, Castilho E. Magnitude e tendência da epidemia de AIDS em municípios brasileiros de 2002-2006. Rev Saúde Pública 2010; 44:430-40.

6. Rodrigues LCB. As vivências da sexualidade de idosos [Dissertação de Mestrado]. Rio Grande: Universidade Federal do Rio Grande; 2008.

7. Rique J, Pinto KM. Perfil epidemiológico dos pacientes HIV/AIDS que abandonaram a terapia retroviral [Monografia de Graduação]. Recife: Faculdade de Enfermagem Nossa Senhora das Graças, Universidade de Pernambuco; 2001.
8. Rodrigues Jr. A, Castilho E. A epidemia de AIDS no Brasil, 1991-2000: descrição espaço-temporal. Rev Soc Bras Med Trop 2004; 37:312-7.

9. Pottes F, Brito A, Gouveia G, Araújo C, Carneiro R. AIDS e envelhecimento: características dos casos com idade igual ou maior que 50 anos em Pernambuco, de 1990 a 2000. Rev Bras Epidemiol 2007; 10:338-51.

10. Toledo L, Maciel E, Rodrigues L, Tristão-Sá R, Fregona G. Características e tendência da AIDS entre idosos no Estado do Espírito Santo. Rev Soc Bras Med Trop 2010; 43:264-7.

11. Perez B, Gasparini S. A vivência do idoso no processo de envelhecer e o HIV/AIDS: uma reconstrução dupla com suas possibilidades e limites. J Bras AIDS 2005; 6:106-9.

12. Gonçalves V, Kerr L, Mota R, Mota J. A Estimativa de subnotificação de casos de AIDS em uma capital do Nordeste. Rev Bras Epidemiol 2008; 11:356-64.

Recebido em 06/Nov/2012

Versão final reapresentada em 10/Mai/2013

Aprovado em 05/Jun/2013 